\title{
Le monastère Saint-Pierre d'Osor (île de Cres) : cinquième campagne d'études archéologiques
}

Morana Čaušević-Bully, Miljenko Jurković, Sébastien Bully et Iva Marić

\section{(2) OpenEdition}

10 Journals

Édition électronique

URL : https://journals.openedition.org/cem/11941

DOI : $10.4000 /$ cem. 11941

ISSN : 1954-3093

Éditeur

Centre d'études médiévales Saint-Germain d'Auxerre

Édition imprimée

Pagination : 103-112

ISSN : 1623-5770

Référence électronique

Morana Čaušević-Bully, Miljenko Jurković, Sébastien Bully et Iva Marić, « Le monastère Saint-Pierre d'Osor (île de Cres) : cinquième campagne d'études archéologiques », Bulletin du centre d'études médiévales d'Auxerre | BUCEMA [En ligne], 15 | 2011, mis en ligne le 26 mars 2013, consulté le 22 septembre 2022. URL : http://journals.openedition.org/cem/11941 ; DOI : https://doi.org/10.4000/ cem. 11941

Ce document a été généré automatiquement le 22 septembre 2022.

\section{(c) (i) (9)}

Creative Commons - Attribution - Pas d'Utilisation Commerciale - Partage dans les Mêmes Conditions 4.0 International - CC BY-NC-SA 4.0

https://creativecommons.org/licenses/by-nc-sa/4.0/ 


\title{
Le monastère Saint-Pierre d'Osor (île de Cres) : cinquième campagne d'études archéologiques
}

\author{
Morana Čaušević-Bully, Miljenko Jurković, Sébastien Bully et Iva Marić
}

1 La cinquième campagne s'est déroulée du 8 mai au 13 juin et du 14 au 19 juillet 2010 . Elle a permis l'achèvement de la fouille du secteur funéraire au chevet de l'église, au terme de quatre chantiers successifs ${ }^{1}$. Nous avons également ouvert deux larges sondages au sud et au nord de l'église - sur la base des résultats de la prospection géophysique réalisée au mois de février 2010 - et débuté la fouille de l'abside centrale de l'église Saint-Pierre. Dans ce secteur, les principales interrogations concernent la datation de la grande église Saint-Pierre et ses origines. C'est sur cette dernière question que la «redécouverte» d'un ex-voto lapidaire, croisée à l'analyse archéologique, permet de proposer de nouvelles hypothèses.

\section{Un premier édifice de culte protobyzantin?}

2 C'est probablement au xIX siècle que deux fragments endommagés d'une architrave (?) portant une inscription chrétienne évergétique ont été découverts à Osor, avant d'être publiée dans le Corpus inscriptionum latinarum [CIL] en $1889^{2}$. Publiée une seconde fois au début $\mathrm{du} \mathrm{xx}^{\mathrm{e}}$ siècle ${ }^{3}$, cette inscription paléochrétienne est restée étonnamment méconnue jusqu'à présent. On ne connaît pas son lieu de dépôt, ni même si elle existe encore aujourd'hui. Mais l'intérêt majeur de l'ex-voto est le lieu de sa découverte: « près de Saint-Pierre », selon la notice du CIL.

3 L'inscription, telle qu'elle est transcrite dans le CIL, est la suivante : DE DONIS DI ET SCI PAVLS S[...] MARIVS TRIBVNOS CON/ CONIOGEN SVAM VV [...]II SVIS VOTOM SVOM SOLVET. Nous en proposons une lecture critique: (croix ?) De donis $D(e) i$ et $s(a n) c(t) i$ Paul $(i), s[(a n) c(t) i$ Petri ?], Marius tribunos con[...] conio[g]en suam $v(i) v(u s)$ [fecit(?) cu]m suis. Votom suom solvet ${ }^{4}$. Les principaux compléments concernent une hypothétique (mais très classique) petite croix au début de la dédicace, ainsi qu'une double titulature à saint Paul et à 
saint Pierre ${ }^{5}$. La lettre D en forme de $\Delta$ et un latin tardif permettent de proposer une datation aux $\mathrm{VI}^{\mathrm{e}}-\mathrm{VII}^{\mathrm{e}}$ siècles et la mention d'un tribun pourrait indiquer la présence d'un commandement byzantin à Osor, contemporaine ou légèrement postérieure à la reconquête justinienne ${ }^{6}$.

4 Il est très vraisemblable que l'architrave inscrite appartenait à un premier édifice de culte, qui se dressait dans le secteur de Saint-Pierre, et peut-être même à son emplacement. Pourrait-on alors envisager que le premier état du mur gouttereau nord de la grande église, tel que l'a reconnu l'analyse archéologique, soit le vestige de cet oratoire ou église? Les montants de porte en marbre remployés dans la façade actuelle de Saint-Pierre - datant des $\mathrm{Xv}^{\mathrm{e}}-\mathrm{xvI} \mathrm{e}^{\mathrm{e}}$ siècles -, dont la modénature évoque l'Antiquité ou l'Antiquité tardive, pourraient également appartenir à cette première phase. Dans l'attente de la fouille de Saint-Pierre, on ne peut en rester qu'au stade d'une hypothèse, mais, qui, si elle se confirmait, permettrait de comprendre les origines de la grande église, faute de pouvoir, jusqu'à présent, en déterminer la datation avec assurance, au début du XI ${ }^{\mathrm{e}}$ siècle ou antérieurement.

\section{La fouille derrière le chevet de l'église (espace III)}

\section{Le cimetière}

5 Cette année a vu l'achèvement de la fouille du cimetière - dans l'emprise du secteur III -, avec le traitement de huit individus, portant à 105 le nombre d'inhumations fouillées depuis 2006, dont 89 dans ce seul secteur. Rappelons que les premières inhumations, majoritairement masculines, sont vraisemblablement monastiques et datent $\mathrm{du}$ $\mathrm{XI}^{\mathrm{e}}$ siècle; dans un second temps, la présence de femmes et d'enfants révèle un recrutement paroissial. Parmi celles-ci, se distinguent quatre sépultures dotées d'éperons que l'on date à partir du XII siècle (fig. 1). Trois de ces inhumations ont été découvertes en 2008, une quatrième l'a été cette année. Le personnage a été inhumé en cercueil : deux boucles de vêtement étaient disposées au niveau de la tête fémorale droite et une troisième reposait entre les coxaux. L'unique éperon, situé au pied droit, est d'un type à mollette à cinq pointes, que l'on date à partir de la seconde moitié du XIII ${ }^{\mathrm{e}}$ siècle. 


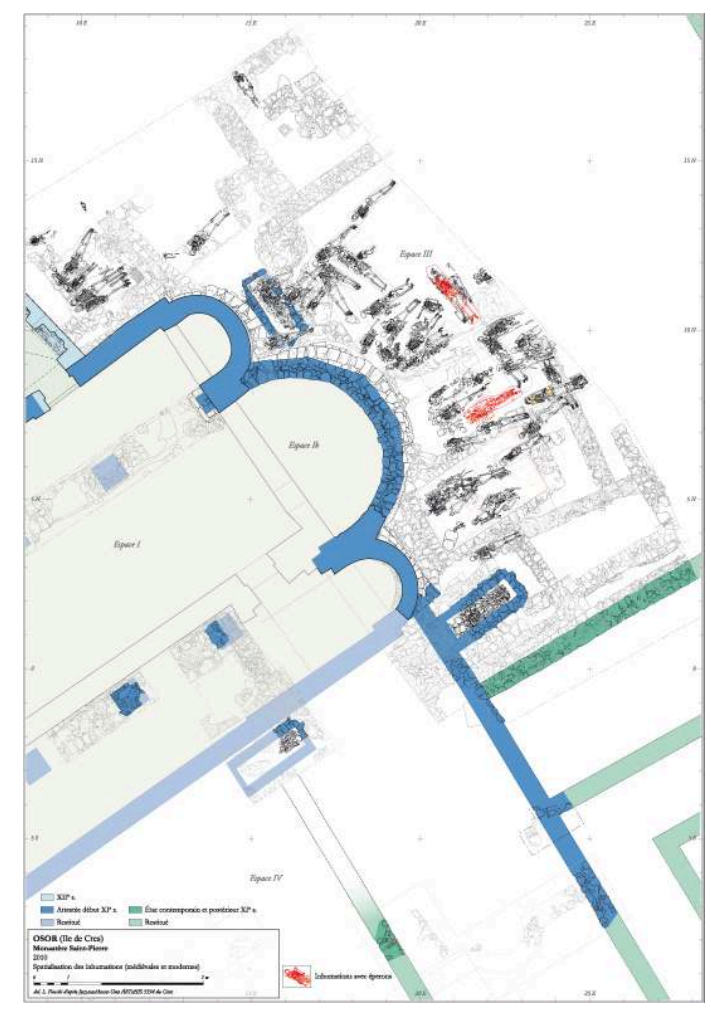

Fig. 1 - Osor, relevé des inhumations au chevet de l'église (dessin L. Fiocchi, D. Vuillermoz et équipe de fouille).

Dans l'attente de l'étude du mobilier archéologique et de la synthèse anthropologique, il apparaît que le cimetière monastique au chevet de l'église abbatiale Saint-Pierre d'Osor a accueilli des inhumations, qui, en dépit de leur simplicité, sont certainement celles de l'aristocratie locale, entre les $\mathrm{XII}^{\mathrm{e}}$ et $\mathrm{XIV}^{\mathrm{e}}-\mathrm{XV}^{\mathrm{e}}$ siècles, comme l'indiquent les quatre tombes de chevaliers.

\section{Les structures d'habitats de l'Antiquité tardive}

7 Le niveau de remblai dans lequel on inhume à partir du $\mathrm{xl}^{\mathrm{e}}$ siècle scelle des structures d'habitats de l'Antiquité tardive, qui semblent perdurer durant le haut Moyen Âge. Mis à part quelques compléments sur le bâtiment $\mathrm{A}$, décrit les années précédentes, c'est essentiellement le bâtiment $\mathrm{E}$ qui a été fouillé. Celui-ci présente des structures fortement arasées, dont il subsiste parfois seulement les tranchées d'épierrement: il occupe le tiers nord du secteur III et se poursuit bien au-delà de la limite de la fouille. Cette unité d'habitation comprend trois états perceptibles à travers des subdivisions internes et des reprises de sols. Le mobilier céramique, les restes de faune et la présence d'un foyer plaident en faveur d'un simple habitat daté du ve siècle, d'après une monnaie de l'empereur d'Orient Arcadius, localisée dans un des premiers niveaux de circulation. Cette datation est confortée par la découverte, dans ces niveaux, de céramique commune au décor de ligne ondulée ${ }^{7}$. 


\section{Sondage nord (espace $\mathrm{VI})$}

8 Un sondage de $5 \times 5 \mathrm{~m}$ a été ouvert au nord de l'église sur la base de l'identification par le radar-sol d'une structure circulaire peu enfouie. Le plan particulier de «l'anomalie radar ", comme sa localisation dans l'axe de l'ouverture latérale de l'église Saint-Pierre, laissait en effet présager une structure inédite, qui s'est révélée être une installation artisanale, que l'on interprète prudemment comme un fouloir à laine. La cuve s'insère dans un environnement déjà occupé par un bâtiment conventuel, adossé contre le flanc nord de l'église abbatiale et le mur de clôture nord (arasé) du monastère.

Le « fouloir » se présente sous la forme d'une maçonnerie grossièrement circulaire en bordure extérieure, d'environ 2,80 $\mathrm{m}$ de diamètre, accueillant une cuve circulaire de $1,40 \mathrm{~m}$ de diamètre par $42 \mathrm{~cm}$ de profondeur (conservée ; fig. 2).

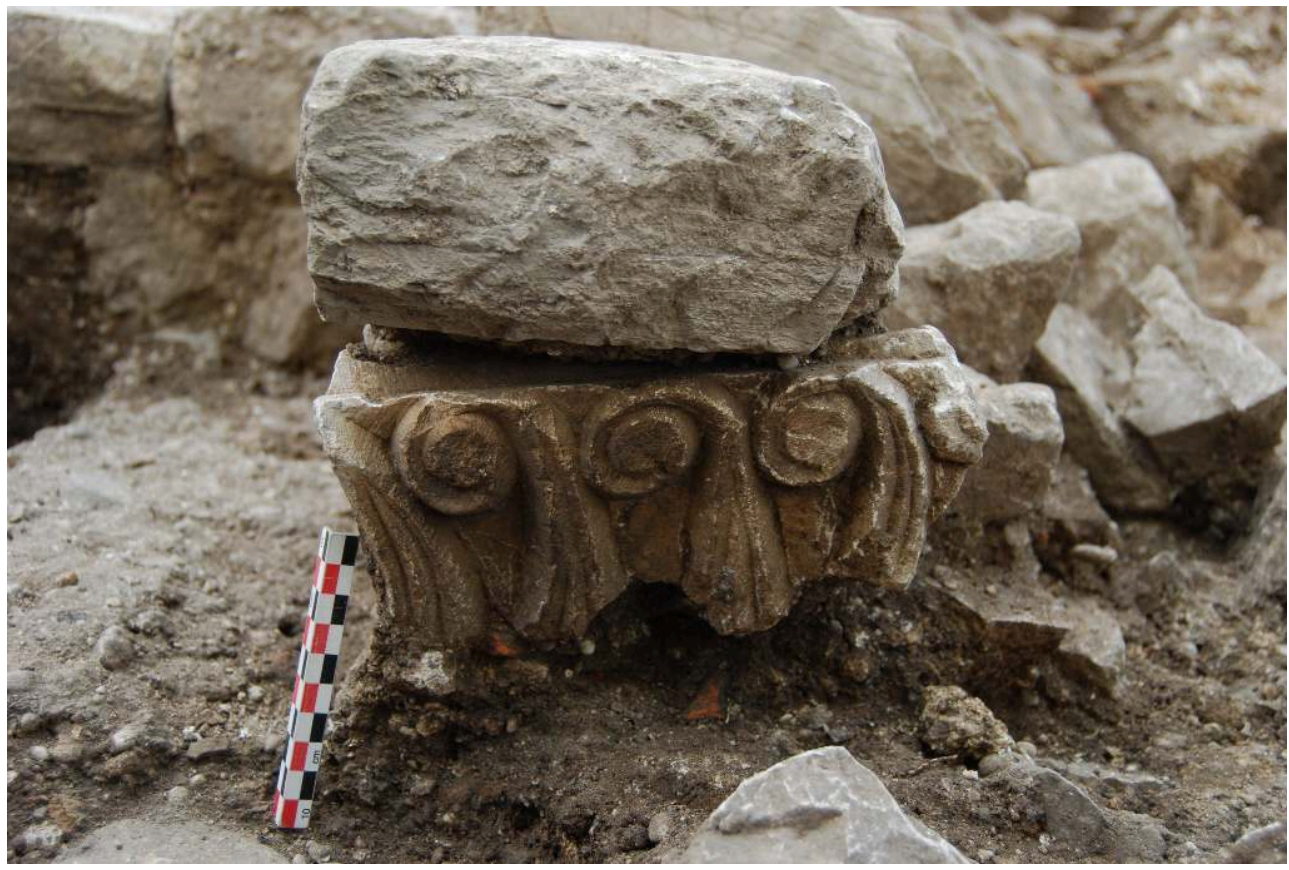

Fig. 2 - Osor, vue depuis l'ouest de la structure circulaire (fouloir?) découverte dans le sondage du secteur VI (cl. B. Bregu).

10 La maçonnerie est composée de blocs non taillés ou seulement équarris, de dimensions irrégulières, liés par un mortier de chaux blanc très compact. La cuve est formée par les fragments d'une vasque de pierre, cassée en huit fragments, avant d'être recomposée et complétée avec de la maçonnerie, afin d'offrir un plus grand diamètre. De fait, la cuve conserve le profil d'une vasque. Le fond de la cuve est constitué par une dalle monolithe à l'aspect poli, avec deux petits creusements ovoïdes en son centre en forme de 8. L'un d'eux accueille une crapaudine métallique avec une mortaise de $3 \mathrm{~cm}$ de diamètre. La dalle de fond est très certainement une épaisse pierre de meule en remploi. Le mortier, entre les fragments de la vasque, se distingue par une couleur légèrement rosée, qui l'apparente à un enduit hydraulique de mauvaise qualité. Les négatifs de blocs arrachés sur l'arase de la cuve laissent supposer que celle-ci était plus profonde. On ne peut donc pas exclure l'existence d'une adduction d'eau, mais il est, en revanche, assuré que la cuve ne possède pas d'évacuation. Sur le flanc est de la structure, la fouille a livré deux trous de poteaux distants de $2,70 \mathrm{~m}$ du centre de la cuve et de 1,20 m entre eux deux. Les poteaux, dont on peut espérer retrouver des 
traces équivalentes sur le bord est de la cuve, auraient pu appartenir à un système de charpenterie recevant un axe vertical fiché dans la crapaudine au fond de la cuve.

Cette installation artisanale pourrait avoir été mise en place dans les dernières décennies du monastère, $a u x v^{e}$ siècle. Cependant, les rares tessons de céramique découverts ne permettent pas de s'en assurer. Une fouille extensive est nécessaire pour comprendre l'évolution de ce secteur au nord du monastère, où l'on a retrouvé également un très grand nombre de scories métalliques, indiquant la proximité d'une forge.

\section{Sondage sud (espace IV)}

Un sondage de 5,4 $\mathrm{m}$ x $3 \mathrm{~m}$ a été ouvert à l'emplacement présumé du contact entre le mur gouttereau sud de l'église et le mur perpendiculaire d'un bâtiment, identifié par A. Mohorovičić lors des premières fouilles en 1953. Il s'agissait d'essayer de déterminer dans quelle mesure le bâtiment - daté alors de l'Antiquité tardive - pouvait avoir été en partie réutilisé dans le monastère, tel que le suggère le plan. Ce secteur a cependant été profondément bouleversé par les travaux anciens, dont on conserve la trace avec une importante tranchée reflétant la méthode employée, qui consistait à suivre les murs. Des fragments de sculptures découverts dans le comblement de l'ancienne tranchée de fouille confirment la rapidité avec laquelle la fouille a été menée.

Trois états ont cependant pu être identifiés. Le premier correspond à une occupation antique marquée par la présence d'une maçonnerie et d'un niveau d'occupation, caractérisé par d'importants restes de consommations de coquillages. Malheureusement, la tranchée de fouille des années 1950 reprend exactement le tracé supposé du mur antique du bâtiment B dessiné sur le plan de Mohorovičić; son existence n'a donc pas pu être confirmée avec assurance dans le sondage, excluant de facto la possibilité d'étudier sa relation avec le mur de l'église. En revanche, le sondage a mis au jour le radier de fondation du mur sud de l'abbatiale, contre lequel on a identifié les vestiges d'une tombe maçonnée, située dans le prolongement du caveau fouillé les années précédentes et bien daté du début du XI ${ }^{\mathrm{e}}$ siècle (fig. 1). Seule la partie orientale de la tombe est conservée sur une longueur d'environ $90 \mathrm{~cm}$; le reste semble avoir été détruit par la tranchée de fouille ancienne, à moins qu'elle ne l'ait déjà été par le mur ouest du bâtiment B identifié par Mohorovičić. Auquel cas, en chronologie relative, le bâtiment pourrait être, tout ou en partie, postérieur à l'église, accréditant ainsi l'hypothèse d'un bâtiment conventuel, intégrant, peut-être, une construction plus ancienne. Le muret sud de la tombe avait déjà été détruit, à un moment où l'on a réduit l'inhumation primaire sous la forme d'un petit ossuaire rejeté au pied. La tombe est construite avec des murets de moellons sans mortier, mais on notera que le petit côté est remployait un beau fragment de sculpture, que l'on peut dater de la fin du VIII ${ }^{\mathrm{e}}$ $\mathrm{x}^{\mathrm{e}}$ siècle d'après son décor dit « de flots » (fig. 3). 


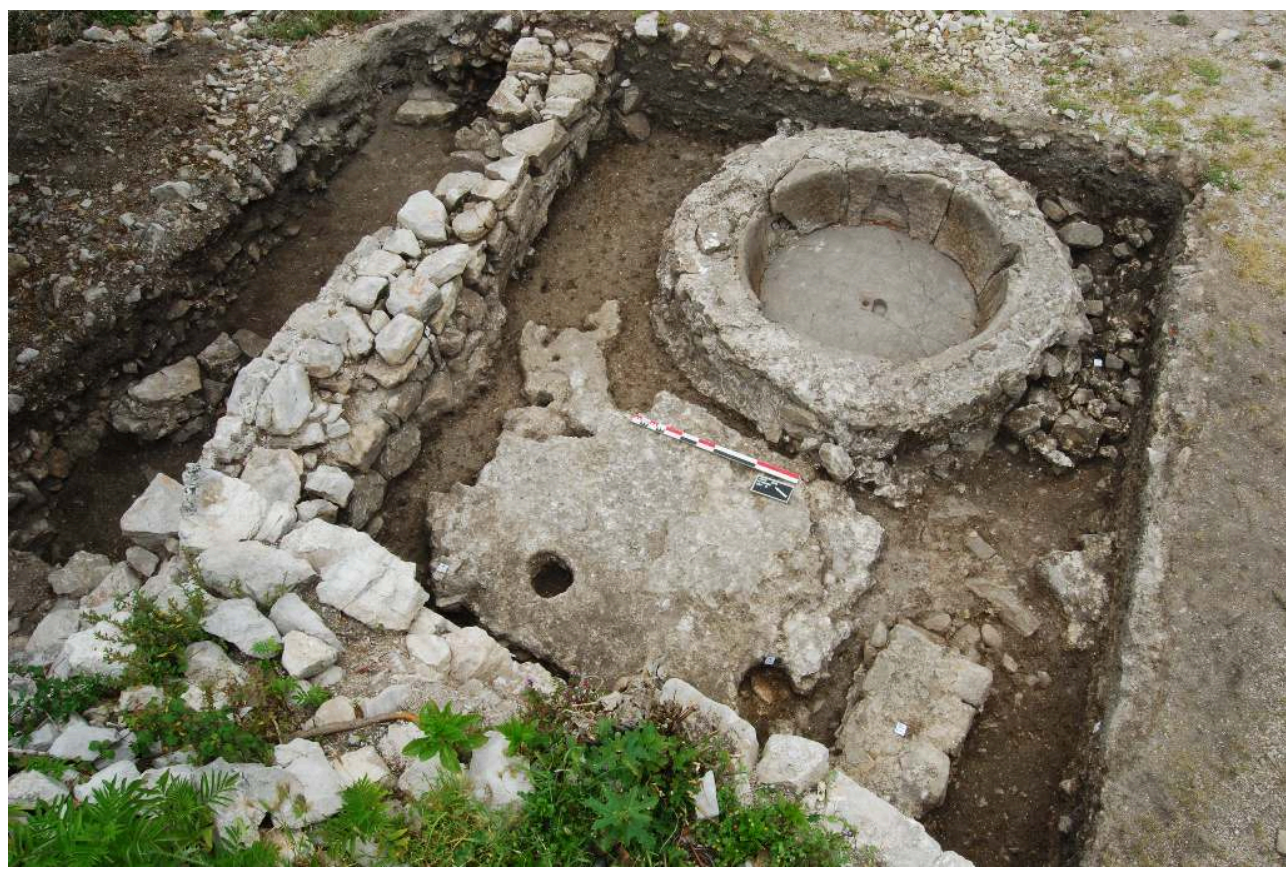

Fig. 3 - Osor, sondage dans le secteur IV ; détail du fragment de sculpture remployé dans la tombe maçonnée (cl. S. Bully).

Ce fragment renvoie à ceux découverts lors de travaux antérieurs et par nous-mêmes dans le comblement de la tranchée de fouilles. Le modelé des motifs d'entrelacs plaide également pour une datation haute, entre les $\mathrm{VIII}^{\mathrm{e}}$ et $\mathrm{x}^{\mathrm{e}}$ siècles. Le sondage a également révélé, contre le mur de l'église actuelle, une puissante maçonnerie $(1.84 \mathrm{~cm})$ fondée sur près de $80 \mathrm{~cm}$ de profondeur. Cette maçonnerie supporte la fondation de la première pile du bas-côté sud, tel un mur stylobate. Mais la différence de méthode constructive, entre le stylobate présumé et le mur gouttereau sud, laisse la porte ouverte à d'autres interprétations, dont celle d'une construction antérieure à la grande église - et peut-être contemporaine de celle identifiée dans le mur nord. Ici aussi, seule l'extension de la fouille dans ce secteur, programmée en 2011, permettra de trancher. Le troisième et dernier état correspond à la rétraction de l'église aux $\mathrm{XV}^{\mathrm{e}}-\mathrm{XVI}^{\mathrm{e}}$ siècles et à la construction d'une tour de clocher contre le nouveau mur sud, dans l'emprise de l'ancien bas-côté sud.

\section{Fouille de l'abside (espace I)}

15 Le chevet à triple abside avait également été découvert par A. Mohorovičić lors de ses travaux en 1953. Le plan publié présentait alors une abside centrale semi-circulaire. Pourtant, le dégagement de l'arase de l'abside, lors de la première campagne en 2006, a révélé que celle-ci était en fait de plan polygonal à l'extérieur, induisant la possibilité d'une datation antérieure au début du $\mathrm{XI}^{\mathrm{e}}$ siècle, par comparaison typologique. Mais audelà de l'analyse formelle du monument, il est rapidement apparu que seules des données archéologiques et archéométriques permettraient de proposer une nouvelle datation de la construction de la grande église. Aussi, la fouille de l'église, et notamment de son abside, outre les données importantes attendues sur les aménagements internes et les états antérieurs, doit, à terme, nous offrir des éléments 
de datation. La première campagne de fouille dans l'abside n'a cependant pas permis de répondre à cette attente.

L'abside mesure $5 \mathrm{~m}$ de diamètre pour une profondeur de 2,84 $\mathrm{m}$ et une épaisseur de mur de $0,60 \mathrm{~m}$. On ne dispose pas de la documentation graphique et photographique des travaux de Mohorovičić, mais il est certain que la restauration de l'arase de la maçonnerie - selon un plan semi-circulaire - date de cette période. Les premiers niveaux de comblement correspondent aux travaux de maçonneries conservatoires et aux remblaiements de la fouille. Mais la stratigraphie du comblement de l'abside a également été fortement perturbée par des creusements de fosses contemporaines, dont l'une pourrait être attribuée à la campagne de Mohorovičić, même s'il n'en relate rien dans ses publications. Il s'agit d'une large fosse aux contours irréguliers présentant un profil en entonnoir : 2,60 m de largeur nord-sud par 1,30 m est-ouest en partie haute, $1,30 \mathrm{~m}$ de diamètre en partie basse par $90 \mathrm{~cm}$ de profondeur. Il est vraisemblable, de par sa situation au centre de l'abside, que le «sondage » de Mohorovičić avait pour objectif de découvrir l'autel. Mais aucun vestige d'autel ou de quelque aménagement que ce soit n'a été repéré au fond de la fosse. La fosse a été creusée, grosso modo, depuis un niveau de sol assez frustre de cailloutis, correspondant au dernier niveau d'occupation de l'abside; il succède à un sol de terre battue subissant un pendage important d'est en ouest et correspondant à un ressaut de fondation intérieur de l'abside. En dépit de sa simplicité, ce sol de terre battue est probablement le dernier niveau de circulation du chœur. La fouille s'est arrêtée sur ce niveau pour cette année, après avoir donc traité essentiellement des perturbations contemporaines.

\section{Conclusion}

17 Avec l'achèvement des études céramologiques et du petit mobilier, la synthèse de la fouille du chevet devra permettre de préciser la chronologie relative entre les structures découvertes et plus particulièrement celles postérieures aux constructions primitives tardo-antiques (bâtiments A et E) : il s'agit en particulier du bassin-citerne, des maçonneries liées à la terre dans le secteur III (bâtiment $\mathrm{D}$ ) et des maçonneries fouillées par Mohorovičić (bâtiment B). Mais il apparaît, d'ores et déjà, que le monastère de Gaudentius s'est établi dans un secteur de la ville urbanisé seulement à partir du IV siècle et dont plusieurs phases de transformations de l'habitat témoignent d'une occupation perdurant encore, vraisemblablement, au haut Moyen Âge. La nature même de l'occupation se précise avec un habitat qui paraît peu structuré, à l'exception peut-être du bâtiment $\mathrm{A}$, dont le portique pouvait être destiné à articuler un espace domestique ou commercial avec un espace public (rue ?) dans sa première phase. La nature des vestiges qu'il nous a été donné d'étudier, plaide, en outre, en faveur d'un simple habitat, excluant l'hypothèse, un temps émise, d'un secteur privilégié de la ville, monumental ou cultuel. Tout au contraire, on a le sentiment d'un secteur périurbain bien qu'à l'intérieur des murs - et tardivement urbanisé.

18 Les premières données archéologiques obtenues lors de la fouille de l'abside et d'une partie du bas-côté sud n'ont pas livré de nouveaux éléments de datation de la construction. En revanche, la redécouverte de l'architrave inscrite et la mise au jour de fragments de sculptures du haut Moyen Âge (d'installations liturgiques ?), conjuguée à la lecture des maçonneries conservées en élévation ou découvertes en sous-sol, ouvrent de nouvelles perspectives sur l'origine et le développement de l'église Saint-Pierre. Ces 
nouvelles perspectives seront plus largement développées avec l'achèvement de la fouille de l'abside et du bas-côté sud, programmé pour la prochaine campagne.

On peut également commencer à esquisser, dans les grandes lignes, la topographie du monastère à la fin du Moyen Âge (fig. 4).

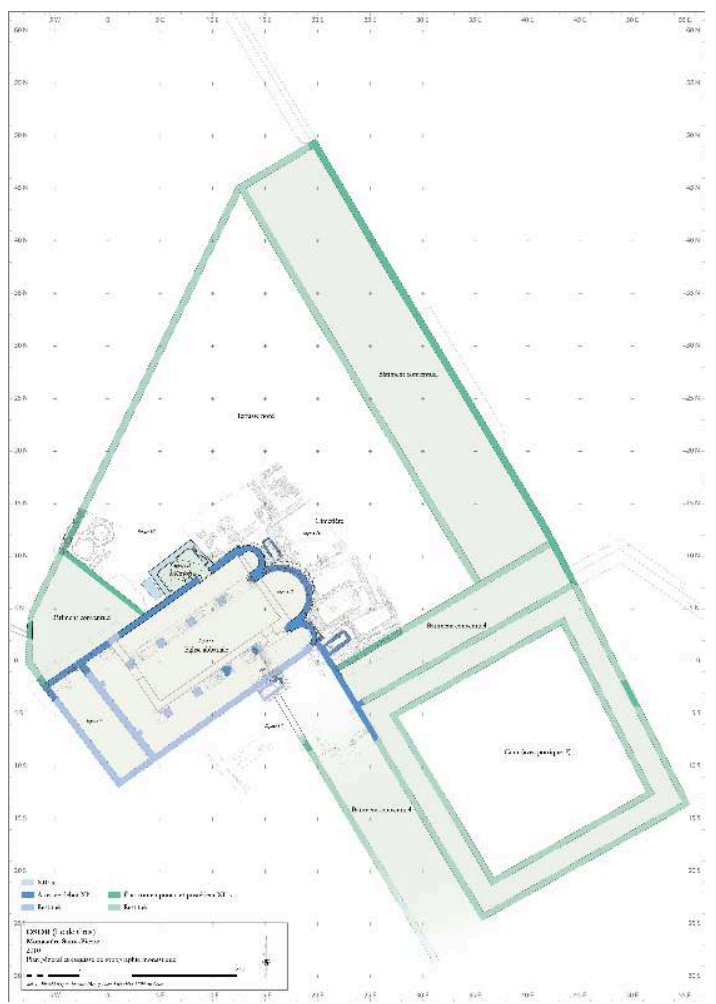

Fig. 4 - Osor, plan général et esquisse de topographie du monastère (dessin L. Fiocchi d'après équipe de fouille).

En effet, rien ne permettait, jusqu'à présent, d'associer l'église abbatiale avec d'autres constructions conventuelles. Désormais, il apparaît qu'un bâtiment était adossé contre son flanc nord et qu'un mur de clôture fermait une terrasse au nord. C'est dans ce secteur que des activités artisanales sont pressenties: forge et fouloir. Le cimetière monastique, au chevet de l'église et occupant une partie de cette terrasse, accueillait également des grands laïcs. D’après la prospection géophysique, le mur de clôture nord se prolongeait vers les hautes murailles, dites « vénitiennes » à l'est. Un rapide examen de l'enceinte indique, d'ores et déjà, plusieurs phases de construction, mais, surtout, il est fort probable qu'un important segment soit en réalité le vestige d'un grand bâtiment conventuel, disposé à l'est de l'église abbatiale, au-delà du cimetière. En 2011, une étude archéologique des élévations d'une partie de cette muraille devrait permettre de s'en assurer. Cette observation va de pair avec les premiers résultats de la prospection géophysique, qui n'avaient pas relevé de structures accréditant, avec assurance, l'existence d'un cloître sur le flanc sud de l'abbatiale. Mais, plus à l'est, se dessine un grand espace quadrangulaire, limité à l'est par le prolongement des murs du bâtiment conventuel hypothétique, que l'on pourrait identifier, sinon à un cloître à la disposition singulière et aux dimensions conséquentes, tout du moins à une cour intérieure du monastère. Cet espace n'a jamais été loti sur les anciens cadastres et est aujourd'hui utilisé comme parking. Une seconde campagne de prospection géophysique sur celui-ci, prévue pour 2011, permettra peut-être de mieux comprendre ce secteur. 


\section{NOTES}

1. Comme chaque année, une version développée de ce compte rendu de fouille sera publiée dans les varia de la revue Hortus artium medievalium. Avec les auteurs de cet article, l'équipe archéologique était formée par Laurent Fiocchi, à qui nous sommes redevables de la plupart des relevés, avec Marie-Laure Bassi et Bruna Bregu, et de leur informatisation. Ont également participé à la fouille : Adeline Barbe, Cyrielle Bille, Thomas Chenal, Sophie Liégard, Alain Fourvel, Karmen Farac, Nirvana Silnović, Maruša Stamać et Ivan Valent. L'opération est conduite par le Centre international de recherches pour l'Antiquité tardive et le Moyen Âge (IRCLAMA) de l'université de Zagreb, l'UMR 5594 Artehis de Dijon, les associations AIPAK et APAHJ. Son financement est assuré par le ministère des Affaires étrangères et Européennes français, les institutions croates (ministère de la Culture et la ville de Mali Lošinj) et la fondation Caritas Veritatis, envers laquelle nous exprimons toute notre gratitude.

2. CIL III, suppl. 2, 10146.

3. E. DIEHL, Inscriptiones latinae christianae veteres [ILCV], Berlin, 1925-1931, nº 1940.

4. En remerciant Françoise Prévot pour ses ajouts à la transcription critique, légèrement différente désormais de celle publiée dans la base de données de Clauss Slaby [http://oraclevm.ku-eichstaett.de:8888/epigr/epigraphik_de].

5. Sur la seule base des vestiges de l'inscription, il est bien évident que la restitution du dernier vocable est conjoncturelle, mais elle ne doit pas être écartée a priori sans examen.

6. L'hypothèse d'un quartier militaire à proximité de Saint-Pierre avait déjà été émise à la suite des fouilles préventives menées sur la «maison Zovko»; cf. J. ČUs-RUKonić, "Arheološka topografija otoka Cresa i Lošinja », in Izdanja HAD- $a$, 1982 ; J. ČUs-RUKONIĆ, « Eastern influence on the religious architecture of the Cres and Lošinj archipelago", in Actes du XIV CIAC, Cité du Vatican/Vienne, 2006, p.317-323; M. ČAUŠEVIĆ-BULLY et J. ČUS-RUKONIć, «La topographie archéologique d'Osor de l'Antiquité au haut Moyen Âge », Histria Antiqua, 16 (2008), p. 262.

7. P. Ruffieux, «Céramique commune de l'Antiquité tardive découverte sur le site de l'église Sainte-Cécile à Guran (Istrie) ", Hortus artium medievalium, 16 (2010), p. 257-266.

\section{INDEX}

Mots-clés : monastère

Index géographique : Croatie (île de Cres)/Osor 